\title{
Efectos post - incendio en bosques de pino del trópico seco de Nicaragua
}

\author{
Luis Miguel Velásquez Benavides ${ }^{1}$ \\ Kenny López Benavides ${ }^{2}$
}

\section{RESUMEN}

La investigación se realizó en la Reserva Natural Serranías de Dipilto y Jalapa. Con el objetivo de evaluar los efectos post-incendio en la riqueza y cobertura de herbáceas y leñosas, en bosque de pino.

Se determinaron tres intensidades de incendio: alta, media, baja y un control (área no incendiada); mediante la cicatriz dejada por el fuego en los fustes de los árboles de pino. Se establecieron parcelas circulares de $314.16 \mathrm{~m} 2$, donde se cuantificó la riqueza y cobertura de especies en tres estratos de la vegetación: arbóreo, arbustivo y herbáceo.

En la alta intensidad se encontraron 22 especies en total. Mientras que en la media y baja intensidad se registraron de 18 especies. En el área no incendiada, se encontraron 12 especies. Es evidente que la mayor riqueza de especies se observó en la alta intensidad, lo cual se debe a la activación de los mecanismos de rebrote y bancos de semilla.

El aumento de la cobertura de especies herbáceas y leñosas es directamente proporcional a las intensidades del incendio, la cual está vinculada a la riqueza de especies.

Palabras claves: Intensidades de incendio, riqueza de especies, cobertura, estratos de la vegetación.

1 UNAN-Managua/FAREM-Estelí. Estación Experimental para el Estudio del Trópico Seco. Correo Electrónico: benavidez90@yahoo.es 2 UNAN-Managua/FAREM-Estelí. Estación Experimental para el Estudio del Trópico Seco. Correro Electrónico: kenny.lb@hotmail.com 


\title{
Fire in pine forests of the dry tropics of Nicaragua-Post effects
}

\author{
Luis Miguel Velásquez Benavidezo ${ }^{1}$ \\ Kenny López Benavides²
}

\begin{abstract}
The research was conducted in the foothills of the Dipilto Nature Reserve and in Jalapa; in order to assess the post-fire effects on the richness and cover of herbaceous and woody pine forest.

Three fire intensities were identified: high, medium, low and a control one (no burned area); by means of the scar left by the fire in the shafts of the pine trees. Circular plots of $314.16 \mathrm{~m} 2$ were established whereby richness and cover in three vegetation strata were quantified: Tree, shrub and herbaceous.
\end{abstract}

In the high intensity a total of 22 species were found. While in the medium and low intensity 18 species were recorded. In the unburned area, 12 species were found. Clearly, the highest species richness was observed in the high intensity, which is due to the activation mechanisms of regrowth and seed banks.

The increased coverage of herbaceous and woody species is directly proportional to the intensity of the fire, which is linked to species richness.

Keywords: fire intensities, species richness, cover, vegetation strata.

1 UNAN-Managua/FAREM-Estelí. Experimental station for the study of the dry tropics. E-mail: benavidez90@yahoo.es 2 UNAN-Managua/FAREM-Estelí. Experimental station for the study of the dry tropics. E-mail: kenny.lb@hotmail.com 


\section{INTRODUCCIÓN}

En ecología debe entenderse el incendio como una perturbación; un fenómeno de origen natural o humano que provoca un cambio importante en el ecosistema. Una definición más precisa de perturbación incluiría aquellos acontecimientos de duración limitada que trastornen la estructura de un ecosistema, comunidad o población y cambien los recursos, la disponibilidad de substrato o el ambiente físico (White \& Pickett, 1985). Toda perturbación es punto de partida de una sucesión ecológica, proceso estructurador de los sistemas naturales y de gran importancia en teoría ecológica, hasta tal punto que (Margalef, 1968) llega a afirmar: «En ecología la sucesión ocupa un lugar similar al que ocupa la evolución en biología general».

Durante las últimas décadas el fenómeno de los incendios forestales ha supuesto una problemática en Nicaragua. Las medidas de contingencia se han limitado al combate del fuego y se ha responsabilizado a la autoridad forestal de la toma de decisiones (FAO, 2001).

Desde finales de la década de los noventa, se han llevado a cabo una serie de actividades políticas que no han ido más allá del simple combate, invirtiendo más recursos humanos y financieros en las medidas de prevención y control. La Defensa Civil del Ejército de Nicaragua en conjunto con asociaciones civiles y diferentes instituciones del Estado, cuentan con un plan de prevención y mitigación de incendios forestales. El resultado de estas actuaciones políticas han sido las llamadas "Agendas de recomendaciones" de lo que debe hacerse para superar la crisis, pero pocas veces se analizan las causas de los incendios, ni las repercusiones sociales y ambientales (SINAPRED, 2007).

A pesar de no existir estudios concluyentes, puede deducirse una correlación entre la vulnerabilidad social y la vulnerabilidad ecológica. Se han realizado estudios en los que se han identificado las causas más frecuentes de los incendios forestales: la caza ilegal, las quemas de maleza (quema de potreros, práctica cultural Silvopastoril, quema de sotobosque en pinares) y las labores agrícolas todas estas de origen antropogénico sumado al aumento de las situaciones climáticas extremas. (INAFOR, 2003). Asimismo, en Nicaragua están ya localizadas las zonas más propensas a incendios forestales. La primera de éstas corresponde al Caribe Norte; seguido de Nueva Segovia, Madriz y Estelí; la tercera a los departamentos de León y Chinandega; la cuarta corresponde al departamento de Rivas; y la última a los departamentos de Matagalpa y Jinotega (SINAPRED, 2007).

En vista de lo anterior, el presente trabajo tuvo como objetivo evaluar los efectos post-incendio en la riqueza y cobertura de herbáceas y leñosas, en bosque de pino. Para tal fin fue necesario, la identificación las especies herbáceas y leñosas recolonizadoras, del área perturbada por las intensidades de incendio. Esto permitirá un nuevo aporte a la información existente sobre la temática. Además facilitará nuevos conocimientos ecológicos sobre los incendios forestales y proporcionará importantes elementos a considerar en la toma de decisiones para la gestión de estos ambientes perturbados por los incendios forestales.

\section{MATERIALES Y MÉTODOS}

El estudio se realizó durante el primer semestre del año 2013. En la Reserva Natural Serranías de Dipilto y Jalapa del municipio de San Fernando, Nueva Segovia, Nicaragua; ubicado entre las coordenadas $13^{\circ} 40^{\prime \prime}$ latitud norte y $86^{\circ} 19^{\prime \prime}$ longitud oeste. Cuenta con elevaciones que oscilan entre los 760 y 1,800 m.s.n.m. El clima es subtropical con tendencia a seco, aumentando la humedad hacia la zona de Jalapa y Murra. La temperatura promedio anual es de $24{ }^{\circ} \mathrm{C}$. La precipitación en invierno varía de 800 a 1,400 mm. El tipo de suelo es de textura franco arenoso. (Plan Ordenamiento Forestal, 2008). 


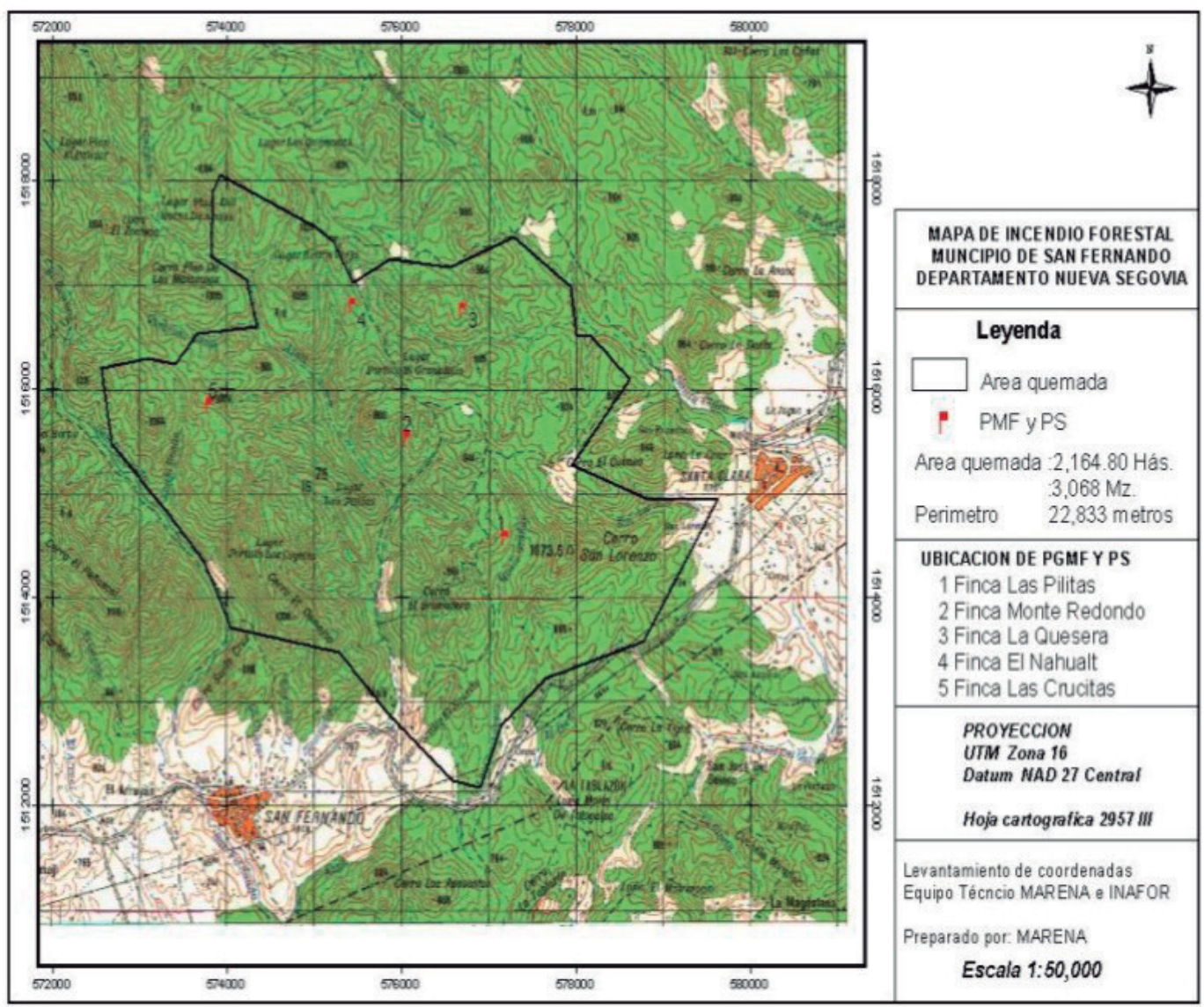

Figura 1: Polígono de Incendio Forestal Municipio San Fernando Departamento Nueva Segovia.

\section{Diseño del muestreo}

Se utilizó el método observacional, el cual consiste en el escaso o nulo control de los factores ambientales. Para este estudio, el factor y sus niveles se definieron en espacio y tiempo. Definiéndose las intensidades con las que quemó el incendio: alta intensidad (AI), media intensidad (MI), baja intensidad (BI) y control o área no quemada $(\mathrm{C})$, establecida fuera del perímetro del área incendiada. Estas se determinaron por las cicatrices dejadas por la acción del incendio en los fustes de los árboles quemados según el método propuesto por (Juárez, 2002).
Para definir el tamaño de las parcelas, se utilizó el método de área mínima de muestreo o curva de acumulación de especies (Braun - Blanquet, 1950 citado por Bautista et al, 2004). En el cual se graficó el número de especies cada cierto tamaño de área. Cuando la curva se estabiliza, el tamaño del área en este momento se considera como el área mínima de muestreo, obteniendo la representatividad de la muestra (Figura 2). 


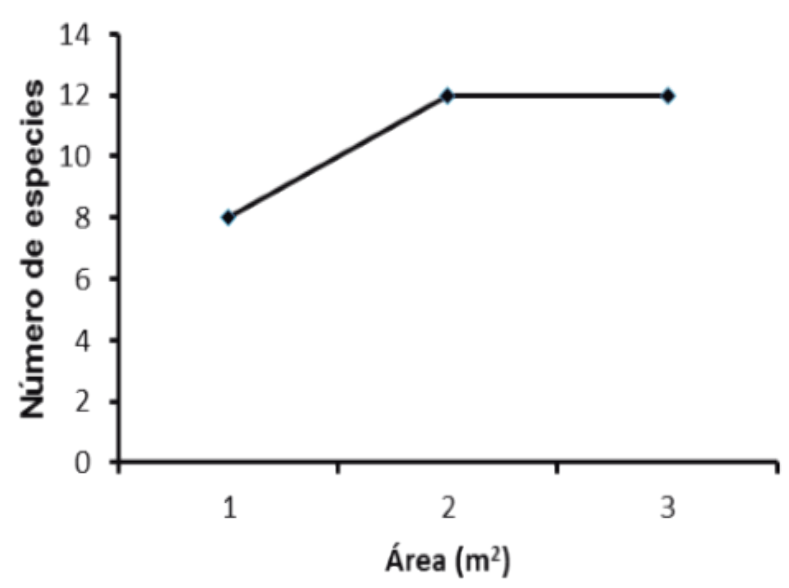

Figura 2: muestra la relación número de especies - área para determinar el área mínima de muestreo.

Donde cada unidad representada en el eje abscisas, equivale a 5 metros de radio elevado al cuadrado y multiplicado por (3.1416). Lo cual significa que los valores 1,2 y 3, corresponden a las áreas 78.54, 314.16, y $706.86 \mathrm{~m} 2$, respectivamente.

Posteriormente se establecieron de 12 parcelas circulares, cada parcela de $314.16 \mathrm{~m} 2$. Se ubicaron tres parcelas por cada intensidad de incendio, donde se muestrearon los tres estratos de la vegetación: herbáceas, arbustivas y leñosas. Se midieron las variables riqueza y cobertura de especies, la cual se estimó mediante la escala de referencia para estimación de cobertura visual de cada una de las especies, según el método propuesto por Braun Blanquet, 1950 (Tabla $1)$.

También se colectaron muestras botánicas fértiles de cada una de las especies para la identificación taxonómica en el Herbario de la Universidad Nacional Autónoma de Nicaragua (UNAN-León).

\begin{tabular}{c}
\hline Braun - Blanquet \\
\hline $1-5 \%$ \\
$6-25 \%$ \\
$26-50 \%$ \\
$51-75 \%$ \\
$76-100 \%$ \\
\hline
\end{tabular}

Tabla 1: Escala de medición de cobertura vegetal.

\section{Análisis estadístico}

Se realizó estadística descriptiva a través de representaciones tabulares y gráficas de barras, sectores y líneas. El software utilizado fue, Excel versión 2010.

\section{RESULTADOS Y DISCUSIÓN}

\section{Especies herbáceas y leñosas recolonizadoras, en las intensidades de incendio.}

Se encontraron 27 especies, agrupadas en 14 familias taxonómicas. De las especies presentes 5 fueron arbóreas, 10 arbustivas y 12 herbáceas. En las tres intensidades de incendio estudiadas, la familia Asteraceae fue el grupo con mayor presencia. Este fenómeno se debe, a que esta familia se caracteriza por su estrategia adaptativa de rebrotar, la cual se activa con el aumento de la intensidad de incendio (Pausas et al, 2009).

Se puede observar que la zona donde hay una mayor riqueza de especies es donde el incendio quemó con alta intensidad. Este hecho se debe a la creación de nuevos hábitats, lo cual implica el aumento de luz solar disponible, menor competitividad por agua y nutrientes y además el aporte de nuevos nutrientes contenidos en las cenizas producto del incendio (DeBano et al., 1998).

La riqueza de especies leñosas y herbáceas fue igual en la baja y media intensidad. Es importante recalcar que esta semejanza entre estas dos zonas es en relación al número total de especies (Figura 3)

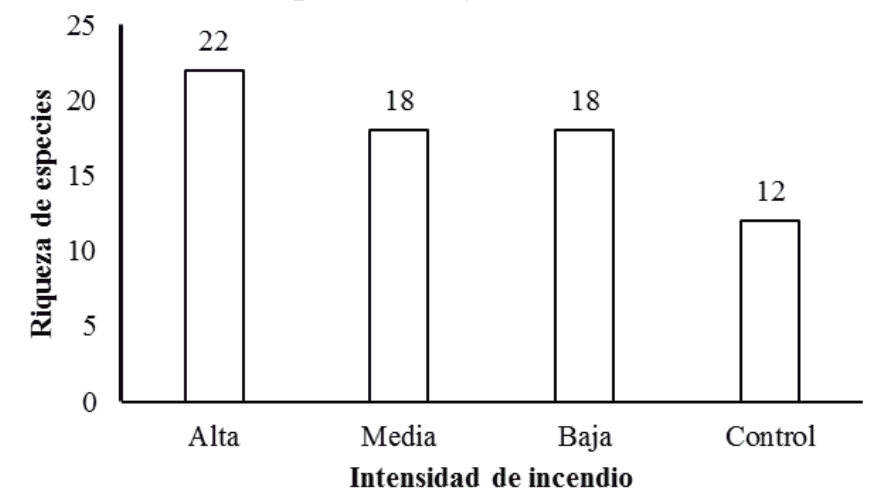

Figura 3: Número de especies encontradas según la intensidad 95 de incendio. 
La riqueza de especies en cada estrato e intensidades de incendio. En la BI y AI de incendio el estrato que registró el mayor número de especies fueron las herbáceas; mientras que la MI el estrato arbustivo alcanzó mayor número de especies. En el área no quemada (C) se observa una disminución considerable de las herbáceas.

En general las herbáceas y arbustivas presentaron mayor riqueza por encima del estrato arbóreo. En el ecosistema de pino, la riqueza de la vegetación post incendio responde de la misma manera que la vegetación de los ecosistemas mediterráneos, la vegetación mediterránea muestra aumentos en la riqueza tras la acción de las altas intensidades de incendio (Coop et al, 2010). Según lo antes mencionado se rechaza la hipótesis nula ya que las intensidades incendio permite que haya un aumento en la riqueza de especies (Figura 4).

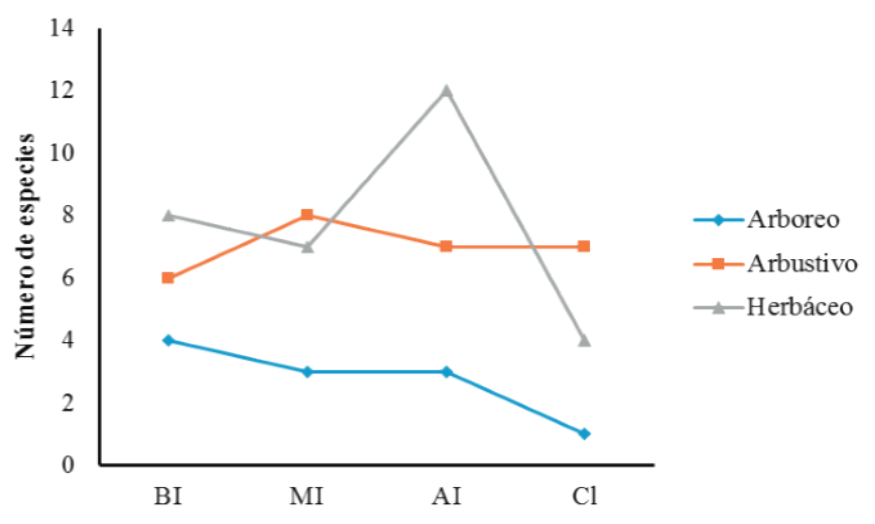

Figura 4: Riqueza de especies según el estrato de la vegetación e intensidad de incendio.

Efecto de las intensidades de incendio, en el porcentaje de cobertura de la vegetación.

El estrato arbóreo ocupa en total un $56.36 \%$ de superficie aproximadamente. Este porcentaje es bajo en comparación a la media intensidad; este comportamiento se debe, a que en estas zonas donde el fuego quemó con baja intensidad hay muy pocos Pinus oocarpa, la especie arbórea predominante en superficie ocupada $31.66 \%$. El incendio del año 2011 no afectó al estrato arbóreo, debido a que la baja intensidad solo afecta el estrato herbáceo y por tanto no hubo regeneración natural, lo cual explica la baja densidad de pinos. Además, es probable según observaciones de campo, que años atrás hayan podido estar gestionados como zona de pastoreo.

El estrato arbustivo recubre en total un $62.53 \%$ del área afectada por el incendio, donde la especie predominante es Mimosa albida con un 26.66\% de cobertura. Estos porcentajes de recubrimiento para el estrato arbustivo son bajos comparados con los de las intensidades mayores. Esto indica que el fuego de baja intensidad no activa ni el banco de semillas, ni los mecanismos de rebrote. Lo antes mencionado coincide con lo planteado por (Pausas et al, 2009), (Molinas y Verdaguer, 1993), que las especies tienen diferentes mecanismos de respuesta al fuego o piroresistencia: rebrotadoras y las no rebrotadoras o germinadoras.

Aun así, esta intensidad, que principalmente afecta al estrato herbáceo, tiene un porcentaje de cobertura 40.96 \% y la especie que obtiene la mayor proporción de superficie ocupada es Synedrella nodiflora con el $20.05 \%$ (Figura 5).

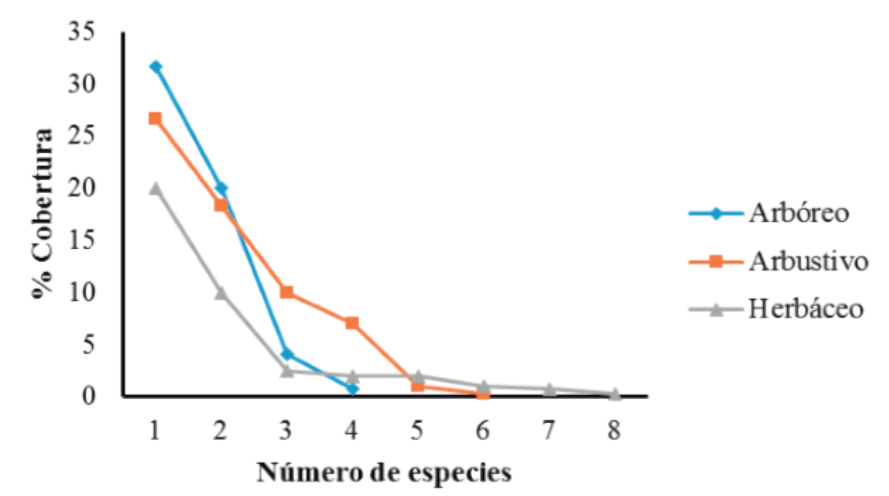

Figura 5: Cobertura de la vegetación por estrato en baja intensidad de incendio.

La superficie arbórea ocupa, el $67.58 \%$ en total, donde la especie Pinus oocarpa, domina un $58.33 \%$ del área. Se podría esperar de estas zonas, que tuvieran aproximadamente la misma superficie arbórea que en 
la baja intensidad pero el gráfico evidencia que no es así. El incendio que tuvo lugar en el año 2003, ya forma parte del pasado pero deja huella en el presente y ahora se puede entender que quemó en este mismo sitio con alta intensidad. Así pues, esta explicación justificaría el aumento de cobertura arbórea y densidad de Pinus oocarpa debido a la regeneración.

En el estrato arbustivo, el 100\% de la superficie está ocupada y la especie predominante fue Mimosa albida con $66.66 \%$. También se encontraron otras especies arbustivas típicas de pinares y que colonizan post incendio pertenecientes a la familia Melastomataceae: Conostegia xalapensis, Tiouchina longifolia y de la familia Malpighiaceae: Byrsonima crassifolia (Tabla 3). Bonilla (2009), encontró estas mismas especies en ecosistema de pino.

El estrato herbáceo se mantiene constante en cuanto a superficie ocupada con un $40.96 \%$, la especie que obtiene el mayor porcentaje de cobertura es Cyathea fulva con $20.66 \%$.

En esta intensidad se pone de manifiesto la capacidad de las especies para rebrotar y germinar después de un incendio forestal. Los bancos de semillas y los mecanismos de rebrote son activados y aproximadamente duplican la superficie arbórea y la arbustiva si se compara con los bajos resultados de la BI. La activación de estas capacidades o características de adaptación (rebrotes, germinación), depende de la intensidad (Aguirre Zhofre, 2000) (Figura 6).

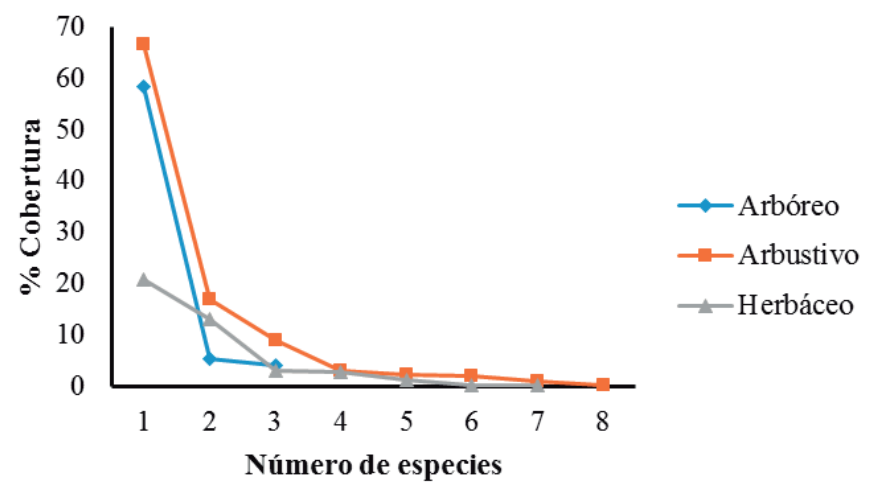

Figura 6: Cobertura de la vegetación por estrato en media intensidad de incendio.
El estrato arbóreo llega a 9.7 \% de ocupación. La virulencia del fuego de alta intensidad provocó una drástica desaparición de todos los ejemplares de Pinus oocarpa. La superficie ocupada por esta especie obtuvo el $8.5 \%$. Es evidente que este valor porcentual es insignificante en comparación con el $31.66 \%$ de la baja intensidad o al $58.33 \%$ de la media intensidad.

El estrato arbustivo alcanza un valor total de cobertura del $69.01 \%$. Este valor no sorprende tanto si comparamos la ocupación de superficie de la zona estudiada que se quemó con media intensidad, que llegó al 100\% de cobertura total. La especie dominante en alta intensidad para este estrato es Mimosa albida con un $56.66 \%$ de superficie ocupada.

En el caso de las herbáceas, ocupan el $81.51 \%$ de la superficie siendo el estrato dominante y la especie que más superficie ocupa es Cyathea fulva con $37 \%$ de cobertura. Es importante prestar atención al aumento de riqueza de las herbáceas en la alta intensidad, debido a que con las nuevas condiciones, aparecerán nuevos nutrientes, habrá la luz solar disponible y menos competitividad y especies que antes no se encontraban en el ecosistema podrán entrar en él. Las herbáceas son las primeras que recubren el suelo tras la acción del incendio (Donoso, 1981).

También aquí se ven activados los bancos de semillas y los mecanismos de rebrote. Estos mecanismos le permiten a las especies colonizar los espacios vacíos dejados por el paso del incendio (Buhk et al, 2006).

Además de activar esos mecanismos de regeneración, la alta intensidad genera las condiciones óptimas en el bosque que permiten que todas estas semillas puedan ser depositadas en el suelo para su posterior desarrollo. El aumento de intensidad deja un sotobosque libre de barreras y las semillas pueden llegar al suelo sin ninguna complicación (Trabaud, 1998). 
En este caso se puede decir que estos mecanismos de adaptación habituales de estas especies son suficientes para garantizar la permanencia de ellas en estos ecosistemas forestales.

En general se evidencia que el aumento de la intensidad de incendio es directamente proporcional a la cobertura de especies, por lo tanto se rechaza la hipótesis nula (Figura 7).
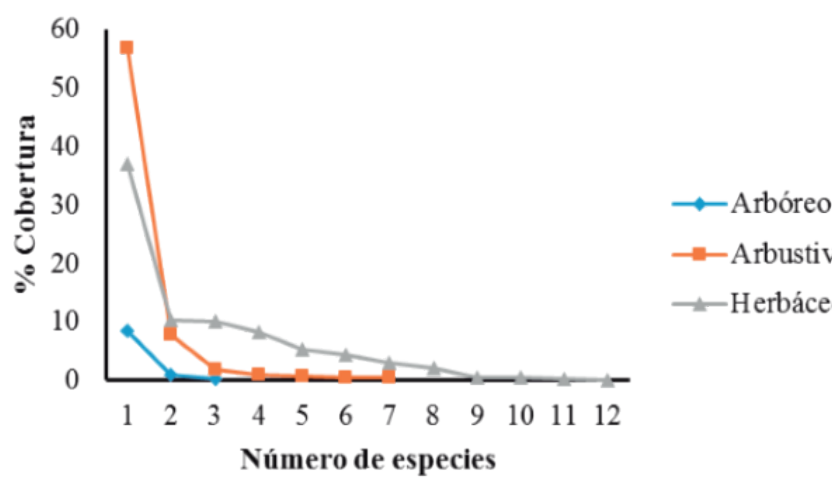

$\rightarrow-$ Arbustivo

$\rightarrow$ Herbáceo

Figura 7: Cobertura de la vegetación por estrato en alta intensidad de incendio.

Se puede observar que el estrato arbóreo ya está establecido en esta zona con un $55 \%$ de superficie ocupada, disminuyendo las oportunidades que tenían las arbustivas y herbáceas para un nuevo establecimiento. Este porcentaje de cobertura corresponde a la especie Pinnus oocarpa.

Con el protagonismo del estrato arbóreo, las especies arbustivas disminuyen bastante pero siguen conservando su riqueza. El porcentaje de superficie para este estrato es $27.6 \%$, la especie que presentó mayor porcentaje de cobertura fue Conostegia xalapensis con $9.6 \%$.

En esta área sufre una importante decaída la riqueza de herbáceas así como la superficie que ocupan de 22.33 $\%$ y la especie dominante fue Verbesina pallens con $13.33 \%$. Esta baja en la riqueza de las herbáceas se debe a que estas alcanzan su mayor frecuencia en $1-5$ años después del incendio y luego reducen su presencia
(Donoso, 1981). Durante los años posteriores a un incendio, la vegetación pasa por distintas etapas en las que la riqueza van cambiando gradualmente, hasta que después de unos años, tienden a estabilizarse dando lugar a una vegetación bastante similar a la presente antes del incendio (Trabaud, 1982) (Figura 8).

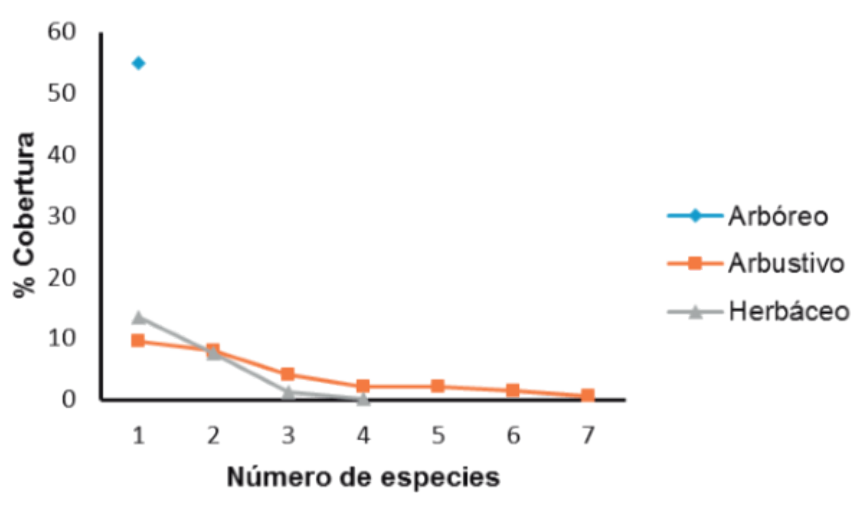

Figura 8: Cobertura de la vegetación por estrato en el control.

El porcentaje de superficie ocupado por la regeneración natural de Pinnus oocarpa en las intensidades de incendio. La alta intensidad presenta el mayor porcentaje de cobertura con $7.33 \%$. Los incendios superficiales y las altas intensidades favorecen la dispersión y liberación de la semilla beneficiando el establecimiento de la regeneración natural (Zendejas, 1971). En estos casos, los individuos adultos de Pinnus oocarpa no resistieron el incendio de alta intensidad y fueron substituidos por otros que nacen de sus semillas y que encuentran un espacio sin competencia, donde llega mucha luz y el suelo es rico en nutrientes (DeBano et al., 1998) (Soto y Rodríguez, 1977) (Figura 9). 


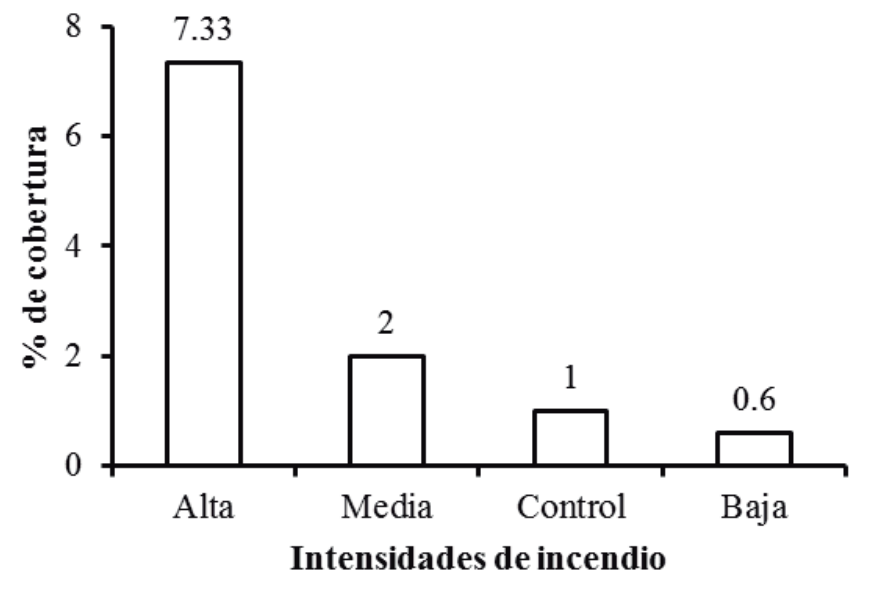

Figura 9: Cobertura de la regeneración natural de Pinnus oocarpa por intensidad.

\section{CONCLUSIONES}

La riqueza aumenta en la alta intensidad en comparación a la media y baja intensidad de incendio.

El recubrimiento de las especies herbáceas y leñosas es mayor a medida que aumenta la intensidad de incendio, por lo tanto también se observan diferencias en cuanto a las intensidades de incendio.

En el control disminuye la riqueza y el recubrimiento en comparación a las áreas afectadas por las intensidades de incendio debido al nivel de recuperación y al dominio del estrato arbóreo en relación al arbustivo y herbáceo.

\section{BIBLIOGRAFÍA}

Altieri Soto, Miguel A.; Rodríguez Muños, Jaime A. (1977). Acción Ecológica del fuego en el matorral natural mediterráneo de Chile, en Rinconada de Maipú. IDIA. Suplemento No 34. P (217 - 222).

Alanis Rodriguez, Eduardo et al. (2010). Efecto de la restauración ecológica post-incendio en la diversidad arbórea del Parque Ecológico Chipinque, México. Madera bosques [online]. vol.16, n.4 [citado 2013-11-19], pp. 39-54<http:// www.scielo.org.mx/o.p?script $=$ sci_t\&pid $=\mathrm{S} 1405$ 04712010000400003\&lng=es\&nrm=iso > . ISSN 1405-0471.

Aguirre Zhofre, M. (2000). Diversidad y composición florística de un área de vegetación disturbada por incendios forestales. $10 \mathrm{p}$. Tesis (Doctor en Ingeniero Forestal) - Herbario Loja, La Paz.

Arianoutsou, M., Beard, J. S., Ferres, L., Folch, R. y Trabaud, L. V. (1993). La vida a les formacions escleròfil•les, en Mediterrànies (Folch, R. coord.). Barcelona, Edit. MAB, UNESCO, 64-109.

Ahlgren I.F. y Ahlgren C.E. (1960). Ecological effects of forest fires. The Botanical Review 26:483-533.

Bautista, F., Delfín, H., Delgado, M., Palacio, J. (2004). Técnicas de Muestreos para Manejadores de para recursos Naturales. Primera Edición. ISBN $970-$ $32-1778$ - 8. Pág. 507.

Begon M, Harper L. (1996). Ecología individuos poblaciones y comunidades. Blackwell science. Localización Biblioteca Luis Angel Arango, Biblioteca departamento de Biología Universidad Nacional sede Bogotá.

Binder, U. (1997). Manual de leguminosas de Nicaragua. Vols. I y II. Programa para la Agricultura Sostenible en Laderas de América Latina (PASOLAC), Escuela de Agricultura y Ganadería de Estelí (EAGE). Estelí, Taller gráfico de los Monjes Agustinos Santa Cruz Estelí. 528 p.

Bonilla. M., Valdez. L., Martinez. L. (2009). Regeneración natural de Pinus tropicalis y vegetación asociada después de un incendio. 6 pág.

Braun-Blanquet, J. (1950). Sociología Vegetal: Estudio de las Comunidades Vegetales. Acme Agency, S. R.L. Buenos Aires, Argentina. 444 pp.

Buhk, C., Götzenberger, L., Wesche, K., Sánchez Gómez, P.yHensen, I. (2006). Post-fire regeneration in a Mediterranean pine forest with historically low fire frequency. Acta Ecologica, 30: 288-298.

Calderón, G.; Rzedowski, J. (2004). Manual de malezas de la región de Salvatierra, Guanajuato. México, Instituto de Ecología, A.C. 316 p. 
Christensen N.L. (1985). Shrubland fire regimes and their evolutionary consequences. En: Pickett S.T.A. y White P.S. Eds. The Ecology of Natural Disturbance and Patch Dynamics, pp. 85-100, Academic Press, Orlando, Florida.

Coop, J.D., Massatti, R.T. y Schoettle, A.W. (2010). Subalpine vegetation pattern three decades after stand-replacing fire: effects of landscape context and topography on plant community composition, tree regeneration, and diversity. Journal of Vegetation Science, 21: 472-487.

Debano, L. F., Neary, D. G. y Ffolliott, P. F. (1998): Fire's effects on ecosystems. New York. John Wiley and Sons.

Donoso Z. (1981). Ecología forestal. El bosque y su medio ambiente. Ed. Univ. Chile.

Font Quer, P. (1953). Diccionario de Botánica. Barcelona, Editorial Labor, S. A. 1244 p

García, J.; MacBryde, B.; Molina, A.; MacBryde, O. (1975). Malezas Prevalentes de América Central. San Salvador, International Plant Protection Center. 162 p. Haidinger, T.L. y Keeley, J.E., (1993). Role of high fire frequency in destruction of mixed chaparral. Madroño. 141 - 147.

Herrarte, J. Ernesto. (2005). Efecto FinancieroAmbiental de los daños ocasionados por los incendios forestales en el periodo comprendido de 1998 al 2003, en el departamento de El Petén.

Holdridge, L.R. (1987). Ecología basada en zonas de vida. Trad. de Humberto Jiménez. San José, IICA, p. 1-44.

Kozlowski T.T., Kramer P.J. y Pallardy S.G. (1991). Fire. En: Kozlowski T.T. Ed. The Physiological Ecology of Woody Plants, pp. 401-424, Academic Press, San Diego.

Kunst, Carlos. (1996). "Introducción a la Ecología de Fuego y Manejo de Fuego Prescripto”, Pág. 134. INTA - UNSE.

Labrada, R.; Caseley, J.C.; Parker, C. (1996). Manejo de malezas para países en desarrollo. (Estudio FAO Producción y Protección Vegetal-120). Roma, FAO. 84 p.
Margalef, R. (1968). Perspectives in ecological theory. University of Chicago Press, Chicago, $111 \mathrm{pp}$.

Mataix, Solera, J. y Guerrero, C. (2007). Efectos de los incendios forestales sobre las propiedades edáficas, en Incendios forestales, suelos y erosión hídrica (Mataix-Solera, J. coord.). Alcoi, Edit. Caja Mediterráneo CEMACAM. 5-40.

Merche B. Bodí. (2012). Efectos de los Incendios forestales en la vegetacion y el suelo en la cuenca Mediterránea. Revisión Bibliografica.

Moody, J. A. y Martin, D. A. (2009). Forest fire effects on geomorphic processes, en Fire effects on soils and restoration strategies (Cerdà, A. y Robichaud, P. R., coord.). Enfield, Edit. Science Publishers, 41-79.

Molinas, M. L. y Verdaguer, D. 1993. «Lignotuber ontogeny in the cork-oak (Quercus suber; Fagaceae). I. Late embryo. " American journal of Botany, n 80.

Moreno J, M., Fernández, F., Vallejo, R., Carbó, E., Bocio, I., Valle, F., Retana, X. y Busquets, I., (1997). Regeneración de la vegetación en zonas quemadas por los grandes incendios de 1994. Estado de la investigación y el desarrollo en protección contra incendios forestales en España. I seminario nacional. 20 - 21 Marzo 1997, Lugo.

Neary, D. G., Klopatek, C., C., Debano, L. F. y Ffolliott, P. F. (1999). Fire effects on belowground sustainability: a review and synthesis. Forest Ecology and Management. $N^{0} 122.51-71$.

Odum, E. P. (1969).The strategy of the ecosystem development Science, 164: 262-270.

Pausas, J. G. y Keeley, J. E. (2009). A burning story: the role of fire in the history of life. BioScience, n $59.593-601$.

Prodon. R., Fons, R. y Athias. Binche. F. (1987). The impact of fire on animal communities in the Mediterranean area, en the role of fire in ecological systems (Tra $\neg$ baud, L. V. coord.). Den Haag, Edit. SPB Academic, 121-157.

Raison, R. J., Khanna, P. K., Jacobsen, K., L. S., Romanya, J. y Serrasolses, I. (2009). Effect of fire 
on forest nutrient cycles, en Fire effects on soils and restoration strategies (Cerdà, A. y Robichaud, P. R., coord.). Enfield, Edit. Science Publishers, 225-256.

Retana J. (1996). Caracteristíques de intensitat i extensio dels incendis. En: Terradas J. Ed. Ecologia del Foc, pp. 59-62, Ediciones Proa, Barcelona, España.

Rodríguez T.D.A. (1996). Incendios Forestales. Universidad Autónoma de Chapingo y Ed. Mundi Prensa, México, D.F.

Ruiz, Jacobo. (2,000). Fuego Factor Ecológico. En Vélez, Ricardo. La defensa contra incendios forestales. McGraw Hill. P.4.1. España.

Salas, J. B. (2002). Biogeografía de Nicaragua. 1ed, Managua: INAFOR, Pág. 548.

Samek, V. (1967). Mejoramiento de los pinos en la práctica forestal. La Habana: Academia de Ciencias de Cuba. 40 p. (Serie forestal, 3).

Sosa C.V., Cedeño O., Rodríguez E., Martínez R. y Raygoza A. (1999). Incendios Forestales. SEGOB y SEMARNAP, México, D.F.

Shakesby, R. A. y DOERR, S. H. (2006). Wildfire as hydrological and geomorphologi $\neg$ cal agent. EarthScience Reviews, no 74, 269-307.

Stevens, W.D.; Ulloa Ulloa, C.; Pool, A.; Montiel, O.M. (eds.). 2001. Flora de Nicaragua. Vols. I, II y III. Missouri Botanical Garden Press. 2666 p.

Toval, H. Nelson y Rueda, P. Ricardo. (2009). Malezas comunes de León, Nicaragua / -- $1^{\mathrm{a}}$ ed. -- Santo Domingo de Heredia, Costa Rica: Instituto Nacional de Biodiversidad, INBio.

Trabaud, L. V. (1990). Fire resistance of Quercus coccifera L. garrigue», en Fire in ecosystem dynamics: Mediterranean and northern perspectives
(Goldammer, J. G. y Jen-kins, M. J. coord.). The Hague, Edit. SPB Academic Publishing, 21-32.

Trabaud, L. (1998). Recuperación y regeneración de ecosistemas mediterráneos incendiados. Serie Geográfica, 7: 37-47.

Trabaud, L. (1982). Effects of past and present fire on the vegetation of the French Mediterranean region. En: Dynamics and Management of the Mediterranean-type

Ecosystems, Conrad, C.E. y Oechel, W.C. (Eds.), pp. 450-457. US Dep. Agric. For. Serv. Gen. Tech. Rep., PSW-58. Symposium, 22-26 junio de 1981, San Diego, CA, USA.

Verzino, Graciela et al. (2005). Impacto de los incendios sobre la diversidad vegetal, sierras de Córdoba, Argentina. Ecol. Apl. [Online]. vol.4, n.1-2 [citado 2013-11-19], pp. 25-34. Disponible en: <http://www.scielo.org.pe/scielo. php? script $=$ sciw4\&lng=es\&nrm=iso $>. \quad$ ISSN 1726-2216.

Vidal, J. J.; COSTANTNO, I. N. (1959): “Iniciación a la Ciencia Forestal". Editorial Salvat.

Viro P.J. (1974). Effects of forest fires on soil. En: Kozlowski T.T. y Ahlgren C.E. Eds. Fires and Ecosystems, pp. 7-45, Academic Press, Nueva York.

White, P.S. \& Pickett, S.T.A. (1985). Natural disturbance and patch dynamics: an introduction. In Pickett, S.T.A. \& White, P.S. (Eds.). The ecology of natural disturbance and patch dynamics. Academic Press, Orlando. Pp. 3-13.

Zendejas E., J.A. (1971). Efecto de las altas temperaturas originadas por el fuego en los conos y semillas de Pinus montezumae y Pinus oocarpa. Tesis profesional. Departamento de bosques, ENA, Chapingo, México. 